\title{
Interactive comment on "Effect of restoration vegetation on the stochasticity of soil erosion in a semi-arid environment” by Ji Zhou et al.
}

\section{Anonymous Referee \#1}

Received and published: 23 August 2016

1. General comments: This manuscript integrated the probabilistic theory and experimental data to systematically assess the stochasticity of soil erosion in different restoration vegetation types. It constructed the OCIRS-Bayes analysis framework to describe the stochasticity of various random events in observed environmental conditions, employed probabilistic approaches to quantify and predict the randomness of soil erosion, and finally used statistic inference theories to discuss the prediction effects of different probabilistic models.

It is a very interesting and attractive study, in particular, from the stochasticity perspectives, it probably supplemented a previous soil erosion studies. However, the language and grammar in this text must be carefully checked to improve the readability of this paper, and other some specific comments or flaws of this manuscript are showed as follows: 
2. Specific comments or flaws: 2.1 In the abstract section Line 32: the change the "erosion random events" into "random erosion events"

2.2 In the introduction section Lines 93-95: This sentence may be deleted, because this paragraph mainly focus on the previous study and missing information about the probabilistic-trait approach application. Lines 93-95 indicated the value of the application of probabilistic-trait on assessing erosion stochasticity, which should put them on the end of the introduction section.

Lines 111-115: These sentences should also be deleted in this paragraph, or be put on the end of the introduction section, because, in the fourth paragraph, the author highlighted the previous research on the effect of vegetation on soil erosion, as well as pointed out the missing information about the effect of vegetation on erosion stochasticity. Lines 111-115 mainly expressed the value of the assessing the effect of vegetation on erosion stochasticity, which should also be finally concluded in the last part of the introduction section.

Line 117-126: This part should be rewrote or restructured. Because, based on the description of missing information, the author should highlight the statement of the purpose and value of this study. I suggest author should put lines 93-95 and lines111-115 together to add into this part, which probably could make the structure of introduction be more logic and clear.

2.3 In the materials and method section In the section 2.3.1 (Lines 178-206), please modified the terminology about the random event expression, in this section the expression of rainfall random event should modified as random rainfall event, which could more clearly express the meaning of those random variables, meanwhile, please carefully check other terminologies involving the probability theory, such as framework, system, to improve the readability of this paper.

2.4 In the result section In the section 3.2 (Lines 314-351), author used two probabilistic approach to analyze the erosive stochasticity in three restoration vegetation types, why

Printer-friendly version

Discussion paper 
did you use this two approaches? What is difference between the two approaches? In particular, the application of Bayes formula on stochasticity analysis was seemed to supply more stochastic information about the soil erosion in different restoration vegetation types, what is the meaning of this exploration?

In the section 3.3 (Lines 356-380), author also used two probabilistic distributions to predict the frequencies of the random runoff and sediment events. Why did you use binomial and Poisson distribution to predict the erosion stochasticity instead of choosing other probabilistic distribution functions?

2.5 In the discussion section In the section, author made a specific explanation of the results, discussed about the difference between binomial and Poisson distribution on predicting the stochasticity of soil erosion by using systematic mathematic proving and statistic inference, furtherly highlighting the value of this study. However, in the section 4.1 (Lines 385-422) author should more clearly pointed out the reason for designing the OCIRS-Bayes framework as well as its effect on the stochastic assessment of soil erosion.

2.6 Figures and tables In the figure 4, (Line 944) total reason? Clerical error. In the table 2 and 3 (Line 847-879) contain some repeated information such as the properties of rainfall events, please check up and simplify the two tables.

Interactive comment on Hydrol. Earth Syst. Sci. Discuss., doi:10.5194/hess-2016-386, 2016. 